\title{
Serial Morphologic Changes on Magnetic Resonance Imaging in a Brainstem Tuberculoma after Antituberculous Treatment
}

\author{
George Kovoor $^{1}$ A. Joe Marshal Leo ${ }^{1}$ Kamble J. Harsha ${ }^{2}$ Akash K. George ${ }^{3}$ \\ ${ }^{1}$ Department of Neurosurgery, Brain \& Spine Centre, Indo-American \\ Hospital, Kerala, India \\ 2 Division of Neuroradiology, Brain \& Spine Centre, Indo-American \\ Address for correspondence Dr. George Kovoor, MS, MCh, \\ Department of Neurosurgery, Indo-American Hospital, Brain \& Spine \\ Hospital, Kerala, India \\ ${ }^{3}$ Department of Research and Development, Brain \& Spine Center, \\ Indo-American Hospital, Kerala, India \\ Indian J Neurosurg 2016;5:59-60.
}

\begin{abstract}
Keywords

- central nervous system tuberculosis

- brainstem tuberculosis

- tuberculoma

- magnetic resonance imaging

Incidence of tuberculosis (TB) is decreasing all over the world due to improved diagnosis and treatment. Though central nervous system TB accounts for $1 \%$ of all TB cases, it carries high mortality and neurologic morbidity. Surgery is difficult when eloquent areas of brain are involved, particularly brainstem. Even with advent of magnetic resonance imaging (MRI) facilities and serologic tests, definitive diagnosis is difficult. We report a case of a 57-year-old-woman with possible brainstem TB, diagnosed based on initial MR images. She was empirically started on anti-TB medications followed by serial repeat MR images at 5,12, and 19 months interval. The initial MR images revealed a thick ring-enhancing lesion, which showed progressive reduction in size, became irregular thin-walled ring-enhancing lesion at 5 months, uniform ring-enhancing lesion at 12 months, and small solid nodular enhancement at 19 months follow-up. There was gradual improvement in neurologic status, and the patient became asymptomatic at the end of 1 year. Characteristic MRI changes over a period favoring adequate response to antituberculosis treatment aids in strengthening presumptive diagnosis of brainstem tuberculoma in resource-poor countries. Along with presumptive diagnosis and timely initiation of medical therapy, periodic MRI follow-up is important in preventing mortality and reducing morbidity.
\end{abstract}

We report the case of a 57-year-old woman presented to our institute with complaints of progressive imbalance while walking, double vision on looking to right lateral aspect, deviation of angle of the mouth toward the left side, inability to close right eyelids completely, and speaking difficulty of 2 weeks duration. Her clinical examination showed horizontal nystagmus with fast component towards right side, right abducens nerve palsy, right lower motor neuron (LMN) facial nerve palsy, staccato speech, and right-side appendicular ataxia. Her erythrocyte sedimentation rate

received

April 7, 2015

accepted

November 23, 2015

published online

February 22, 2016
(ESR) was $57 \mathrm{~mm} / \mathrm{h}$ and chest X-ray was normal. Magnetic resonance (MR) images revealed a large thick irregularwalled ring-enhancing lesion in the right hemipons (-Fig. 1A-C). MR spectroscopy from the lesion revealed large lipid peak without significant choline elevation. Findings were consistent with inflammatory etiology, possibly tuberculoma.

The patient was started on antituberculosis medications and periodic follow-up MR images were obtained. The initial thick-walled intensely enhancing ring lesion became thin
DOI http://dx.doi.org/

10.1055/s-0036-1572366. ISSN 2277-954X.
(C) 2016 Neurological Surgeons' Society of India
License terms

(잉 (1) $\Theta \circledast$ 


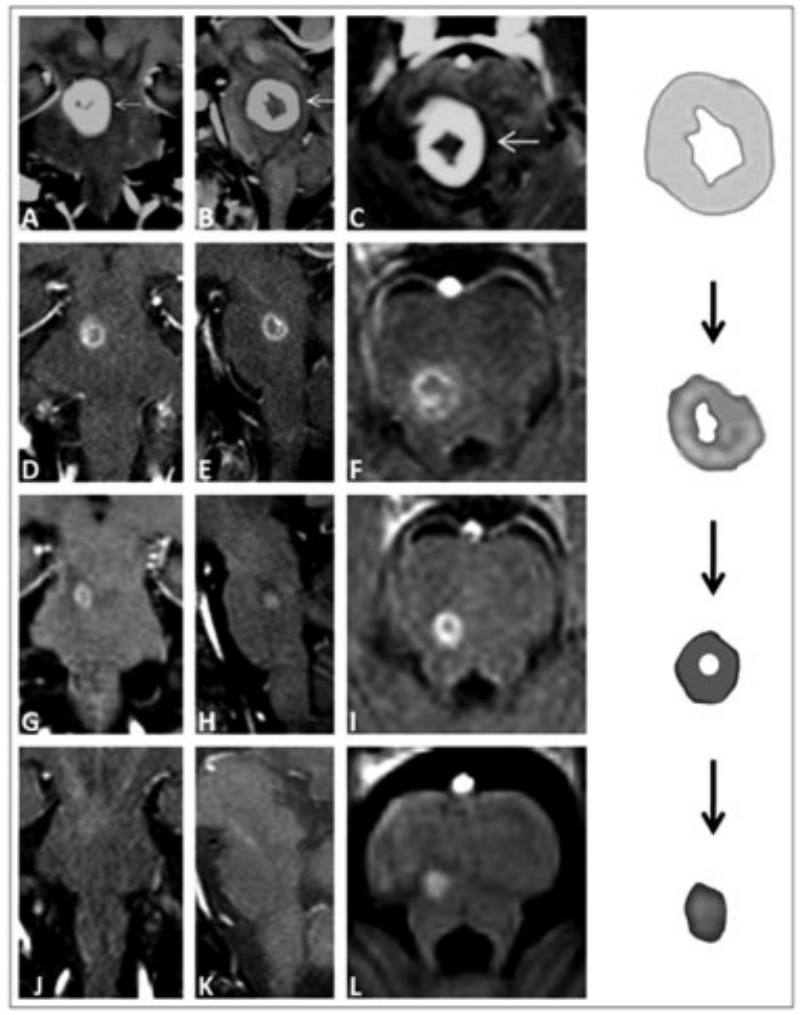

Fig. 1 Serial MRI coronal, sagittal, axial postcontrast T1W images and corresponding diagrammatic representation of resolving lesion. (A-C) Initial images revealed a large thick irregular-walled ringenhancing lesion. (D-F) At 5 months, significant reduction in size and wall thickness. (G-I) At 12 months, further reduced size, loss of wall irregularity of ring-enhancing lesion. (J-L) At 19 months, delayed small solid contrast enhancement of lesion.

irregular-walled mildly enhancing lesion at 5 months (-Fig. 1D-F). MR images after 12 months showed further reduction in size and loss of wall irregularity of ringenhancing lesion (-Fig. 1G-I). The patient became asymptomatic at the end of 12 months. The 19-month MR image revealed conversion of previously ring-enhancing lesion into delayed solid-enhancing lesion, possibly secondary to clearance of central necrotic material, minimal gliosis indicative of minimal damage to brainstem structures (-Fig. 1J-L). Though a large $1.5-\mathrm{cm}$ lesion in pivotal area of brainstem is expected to heal with extensive damage, the early initiation of specific therapy along with steroid administration has minimized the damage.

Central nervous system tuberculoma diagnosis is challenging, as many patients have no evidence of pulmonary tuberculosis (TB). This is further complicated when it is in the brainstem, which makes it unamenable to biopsy. Brainstem being an uncommon location consists 2.5 to $8 \%$ of all intracranial lesions, ${ }^{1}$ which is the $1 \%$ among all active TB cases. $^{2}$ Clinical and serologic response to antituberculosis therapy with serial MRI analysis can point toward the diagnosis almost certain as the computed tomography (CT) and MRI have revolutionized the diagnosis of intracranial tuberculomas. ${ }^{3,4}$ This case report emphasizes characteristic serial morphologic changes on MRI in a brainstem tuberculoma over a period of time favoring adequate response to antituberculosis treatment, and aids in strengthening presumptive diagnosis of brainstem tuberculoma in resource poor countries. Along with presumptive diagnosis, timely initiation of medical therapy and periodic MRI follow-up are important in preventing mortality and reducing morbidity.

\section{Conflict of Interest}

None.

Funding

None.

\section{References}

1 Kumar R, Jain R, Kaur A, Chhabra DK. Brain stem tuberculosis in children. Br J Neurosurg 2000;14(4):356-361

2 Bas NS, Güzey FK, Emel E, Alatas I, Sel B. Paradoxical intracranial tuberculoma requiring surgical treatment. Pediatr Neurosurg 2005;41(4):201-205

3 Gupta RK, Jena A, Singh AK, Sharma A, Puri V, Gupta M. Role of magnetic resonance (MR) in the diagnosis and management of intracranial tuberculomas. Clin Radiol 1990;41(2):120-127

4 Gupta RK, Jena A, Sharma A, Guha DK, Khushu S, Gupta AK. MR imaging of intracranial tuberculomas. J Comput Assist Tomogr $1988 ; 12(2): 280-285$ 